\title{
Strategies of imaging after orthopedic surgery
}

\author{
A. Reginelli ${ }^{1}$ M. Zappia ${ }^{2}$ A. Barile ${ }^{3}$ L. Brunese ${ }^{2}$
}

Received: 11 October 2016/ Accepted: 15 January 2017/Published online: 24 February 2017

(C) Istituto Ortopedico Rizzoli 2017

In recent years, the development of many surgical techniques and the substantial increase in number of surgical procedures in orthopedics have led to a consequent increase in demand for postoperative imaging examinations of the joints.

Performing and interpreting MRI or CT of the joints in patients after surgery is a difficult task. The normal anatomic features are distorted by the surgical alterations as well as the artifacts that result from metal and other materials used in the surgical procedures.

To date, there is much confusion in the interpretation of the images of the patients, because in most cases the radiologist is not aware of the surgical procedure performed, but even more because the result of postoperative imaging is often discrepant with the clinical course.
Thus, it happens that in patients with a perfect outcome of surgery, the MRI gives pathological results, leading to unnecessary and costly lawsuits; on the other hand, often the orthopedic surgeons do not find an adequate answer to their questions in imaging of patients with real postsurgical problems.

The purpose of this review is to outline the elements of semiotics useful to the radiologist to: recognize and evaluate the surgical method used; identify and evaluate the placement of the current surgical devices; understand the normal postsurgical changes, in order not to confuse them with pathological alterations; and compare the imaging changes with the surgery technique and the time elapsed by it.
A. Reginelli

alfonsoreginelli@hotmail.com

L. Brunese

lucabrunese@libero.it

1 Department of Internal Clinical and Experimental Medicine and Surgery, Second University of Naples, Caserta, Italy

2 Department of Medicine and Health Science "V. Tiberio", University of Molise, Campobasso, Italy

3 Department of Biotechnologies and Applied Clinical Sciences, University of L'Aquila, L'Aquila, Italy 